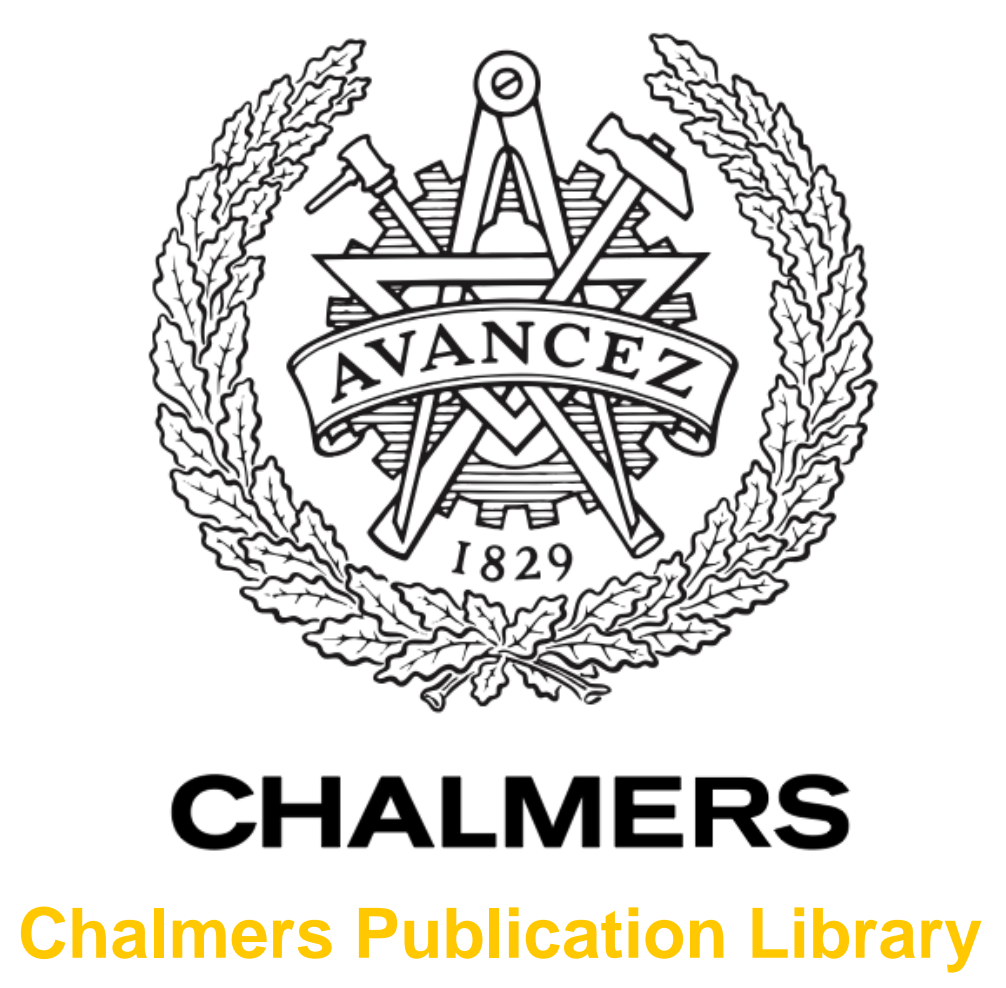

Modeling system throughput of single and multi-port wireless LTE devices

This document has been downloaded from Chalmers Publication Library (CPL). It is the author's version of a work that was accepted for publication in:

2012 IEEE International Symposium on Antennas and Propagation, Chicago, USA, July 814, 2012 (ISSN: 1522-3965)

Citation for the published paper:

Hussain, A. ; Kildal, P. ; Durisi, G. (2012) "Modeling system throughput of single and multiport wireless LTE devices". 2012 IEEE International Symposium on Antennas and

Propagation, Chicago, USA, July 8-14, 2012

http://dx.doi.org/10.1109/APS.2012.6348700

Downloaded from: http://publications.lib.chalmers.se/publication/163385

Notice: Changes introduced as a result of publishing processes such as copy-editing and formatting may not be reflected in this document. For a definitive version of this work, please refer to the published source. Please note that access to the published version might require a subscription.

Chalmers Publication Library (CPL) offers the possibility of retrieving research publications produced at Chalmers University of Technology. It covers all types of publications: articles, dissertations, licentiate theses, masters theses, conference papers, reports etc. Since 2006 it is the official tool for Chalmers official publication statistics. To ensure that Chalmers research results are disseminated as widely as possible, an Open Access Policy has been adopted.

The CPL service is administrated and maintained by Chalmers Library. 


\section{Modeling System Throughput of Single and Multi-port Wireless LTE Devices}

\author{
Ahmed Hussain, Per-Simon Kildal \\ Antenna Group, Dept. of Signals \& Systems \\ Chalmers University of Technology \\ Gothenburg, Sweden \\ ahmed.hussain@chalmers.se
}

\author{
Giuseppe Durisi \\ Communication Group, Dept. of Signals \& Systems \\ Chalmers University of Technology \\ Gothenburg, Sweden
}

\begin{abstract}
We have recently introduced a simple theoretical model for estimating the relative throughput of LTE devices. We validated the model through measurements performed in reverberation chamber for $1 \times 2$ SIMO with 90 ns channel delay spread. In the present paper, we extend our previous study to compare the frequency diversity offered by different channel delay spreads emulated in reverberation chamber for SISO and $1 \times 2$ SIMO configurations.
\end{abstract}

\section{INTRODUCTION}

Long Term Evolution (LTE) is the latest wireless communication standard after WCDMA and GSM. The 3GPP LTE standard [1] includes orthogonal frequency division multiplexing (OFDM) and multiple-input multiple-output (MIMO) technology, which makes LTE unique in terms of high reliability, which is obtained through the exploitation of both spatial and frequency diversity. The idea behind diversity is to ensure that the transmitted data reach the receiver through independent signal paths [2]. This makes a system using diversity techniques robust against strong reductions in the signal strength due to channel fading.

Reverberation chambers can emulate rich isotropic multipath environments suitable for testing of small antennas in wireless devices [3]. It has recently also been shown that MIMO-LTE throughput, which is one of the key performance metrics for wireless terminals, can be easily measured in a reverberation chamber [4]. Furthermore, a simple throughput model, based on the concept of digital threshold receiver [4] has been proposed and the corresponding predicted throughput curves shown to agree with the measured throughput curves.

In this paper, we use the OFDM frequency diversity formula in [4] to evaluate the dependency of the throughput on the coherence bandwidth of the wireless fading channels generated inside the reverberation chamber. We consider SISO and $1 \times 2$ SIMO diversity configurations.

\section{THE SIMPLE THROUGHPUT MODEL}

The throughput model proposed in [4] is motivated by the observation that the error-rate curve of wireless transceivers equipped with modern error correcting codes (see, e.g, [5]) and operating over an additive white Gaussian noise (AWGN) channel changes very steeply from about $100 \%$ error-rate to about $0 \%$ error-rate as the average received power $P_{\mathrm{r}}$ exceeds a certain threshold $P_{\text {th }}$. The relationship between error-rate $\varepsilon$ and throughput $T$ can be expressed as:

$$
T=R \times(1-\varepsilon) .
$$

Here, $R$ is the maximum achievable data-rate for a given ideal system. We have $T=0$ when $\varepsilon=1$ (i.e., $100 \%$ error-rate) and $T=R$ when $\varepsilon=0$ (i.e., $0 \%$ error-rate). The value of $P_{\text {th }}$ depends not only on the modulation and coding schemes adopted but also on the hardware components used in the transceiver, such as LNAs, mixers, power amplifiers, antennas, etc. Hence, $P_{\mathrm{th}}$ is not only configuration-specific but also device-specific. As shown in [4], $P_{\text {th }}$ can be characterized by means of conductive measurements where the transmit power is increased until a sharp change in the system throughput is observed.

When Rayleigh fading is present in the communication link, the throughput curves exhibit a much smoother and slower change from $100 \%$ to $0 \%$ error-rate i.e. from minimum to maximum throughput. As shown in [4], this change can be accurately described by the cumulative density function (CDF) of the received power. The CDF is not only a function of the antenna diversity configuration (SISO or SIMO) but also a function of the amount of frequency diversity offered by the fading channels (longer or shorter delay spreads).

\section{MEASUREMENT SETUP}

The average path loss in the reverberation chamber is measured with a vector network analyzer (VNA) by using high-efficiency reference antenna inside the reverberation chamber. As shown in Figure 1 (left), the LTE base station and the wireless device are connected through cables for the conductive measurements used to determine $P_{\mathrm{th}}$; the loss due to these cables is calibrated out. To conduct over-the-air (OTA) measurements, both the LTE base station and the wireless device are connected to antennas residing inside the reverberation chamber; the wireless device and the LTE base station are situated outside the chamber in shielded boxes, see Figure 1 (right). Cable losses, internal losses of the antennas and the average path loss inside reverberation chamber are measured and calibrated out. To generate wireless channels with different delay spreads [6], the reverberation chamber is loaded with different number of absorbers thereby changing the path loss inside the reverberation chamber. Therefore, separate calibration measurements are performed for each loading case of the reverberation chamber to ensure low uncertainty. The loads also increase the average Rician Kfactor [7] in the chamber, and thereby increase the measurement uncertainty, but it is still quite acceptable [8]. 
A Rhode \& Schwarz's CMW500 LTE base station simulator and a Huawei's LTE USB dongle are used as measurement instrument and device-under-test (DUT) respectively. The uplink (UL) and downlink (DL) modulation schemes used are QPSK and 64-QAM respectively. The measurements are performed using Band 7 (i.e., DL channel number 3100, DL frequency $2655 \mathrm{MHz}$ ) and $10 \mathrm{MHz}$ bandwidth. We average 100 measurement samples for each power-level.

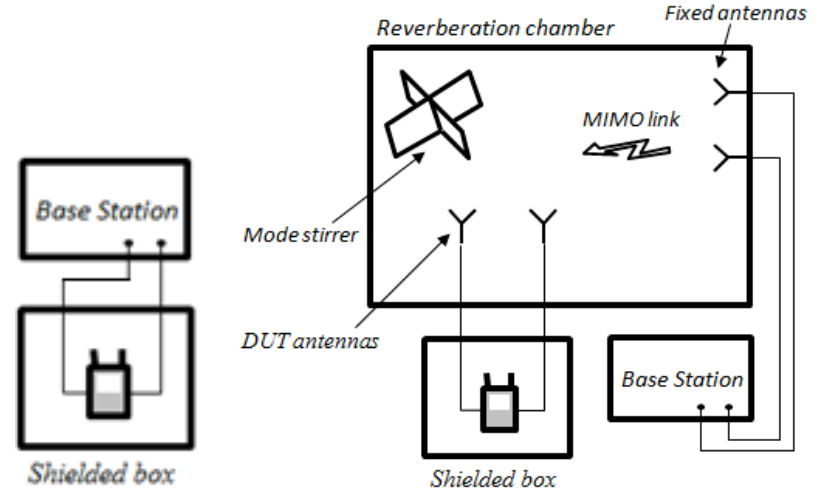

Figure 1: Conductive (left) and OTA Measurement Setup (right)

\section{RESUlTS AND CONCLUSION}

The throughput was measured for the delay spread of 35ns, $85 \mathrm{~ns}$, and 215ns, corresponding to coherence bandwidth $B_{c}$ of approximately $8 \mathrm{MHz}, 3 \mathrm{MHz}$, and $1.3 \mathrm{MHz}$, respectively, for SISO and $1 \times 2$ SIMO configurations. The measurement results with 35ns, 85ns, and 215ns delay spread cases are plotted against the theoretical results obtained using the simple model devised in [4] corresponding to 1,2 , and 3 independent number of frequency diversity channels $N_{f d}$ respectively, with a constant $C=0.45$. The agreement is good. We expected a larger diversity gain for this setting. It seems that the OFDM implementation under consideration does not exploit all the frequency diversity provided by the channel. From SISOconductive measurements, we get $P_{t h}=-77.8 \mathrm{dBm}$. Note that we have adjusted the threshold value slightly as shown in the legends to align the curves, and verify whether their slopes match. The good agreement is in line with similar results observed when measuring passive devices, including comparisons with measurements in anechoic chambers [9].

\section{ACKNOWLEDGEMENT}

This work has been supported in part by the Swedish Governmental Agency for Innovation Systems (VINNOVA) within the VINN Excellence Center Chase, and by the Swedish Strategic Research Foundation (SSF) within the Chalmers Microwave Antenna Systems Research Center CHARMANT.

\section{REFERENCES}

[1] http://www.3gpp.org/

[2] D. N. C. Tse and P. Viswanath, Fundamentals of Wireless Communications, Cambridge, U.K.: Cambridge Univ. Press, 2005.

[3] P. S. Kildal and K. Rosengren, "Correlation and capacity of MIMO systems and mutual coupling, radiation efficiency, and diversity gain of their antennas: simulations and measurements in a reverberation chamber," IEEE Communications Magazine, vol. 42, pp. 104-112, 2004.

[4] P. S. Kildal, A. Hussain, X. Chen, C. Orlenius, A. Skårbratt, J. Åsberg, T. Svensson, and T. Eriksson, "Threshold Receiver Model for Throughput of Wireless Devices with MIMO and Frequency Diversity Measured in Reverberation Chamber," IEEE Antennas and Propagation Wireless Letters, vol. 10, pp. 1201-1204, October 2011.

[5] T. J. Richardson and R. Urbanke, Modern Coding Theory. Cambridge, U.K.: Cambridge Univ. Press, 2008

[6] X. Chen, P. S. Kildal, C. Orlenius, and J. Carlsson, "Channel Sounding of Loaded Reverberation Chamber for Over-the-Air Testing of Wireless Devices: Coherence Bandwidth Versus Average Mode Bandwidth and Delay Spread," Antennas and Wireless Propagation Letters, IEEE, vol. 8, pp. 678-681, 2009.

[7] X. Chen, P. S. Kildal, and S. H. Lai, "Estimation of Average Rician KFactor and Average Mode Bandwidth in Loaded Reverberation Chamber," Antennas and Wireless Propagation Letters, IEEE, vol. 10, pp. 1437-1440, 2011.

[8] P.-S. Kildal, X. Chen, C. Orlenius, "Characterization of Reverberation Chambers for OTA Measurements of Wireless Devices: Formulation of Channel Matrix and Uncertainty," submitted to IEEE Transactions on Antennas and Propagation, September 2011.

[9] X. Chen, P. S. Kildal, J. Carlsson, and J. Yang, "Comparison of Ergodic Capacities From Wideband MIMO Antenna Measurements in Reverberation Chamber and Anechoic Chamber," IEEE Antennas and Wireless Propagation Letters, vol. 10, pp. 446-449, 2011.
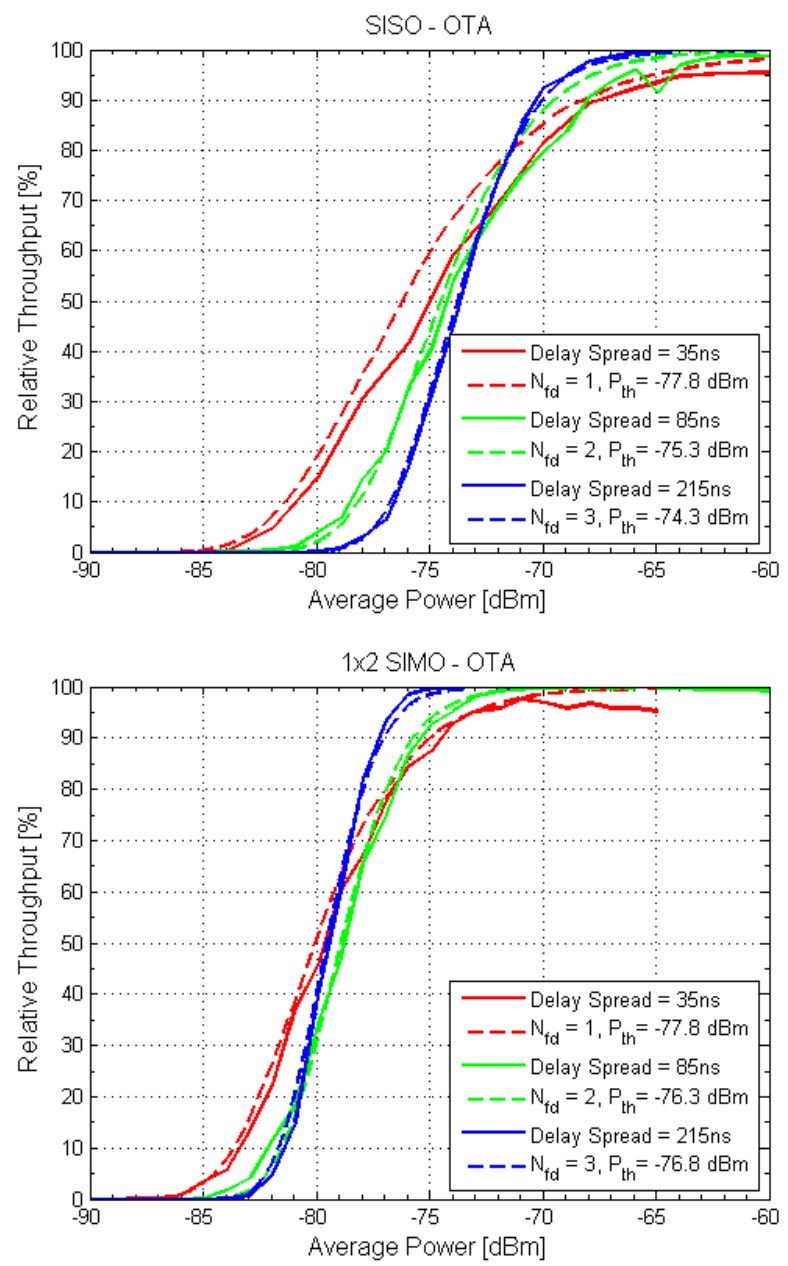

Figure 2: Relative OTA Throughput of LTE device using different delay spreads - Measurements and Model 